Supporting Information

\title{
Aromatic Core Modified Decaphyrins With Largest Two-photon Absorption Cross sections: Syntheses and Characterization
}

\author{
Harapriya Rath $^{\dagger}$, Viswanathan Prabhuraja ${ }^{\dagger}$, Tavarekere $\mathrm{K}$. \\ Chandrashekar ${ }^{\star} \S$, Amit $\mathrm{Nag}^{\dagger}$, Debabrata Goswami ${ }^{\dagger}$, Bhawani S. \\ Joshi $i^{¥}$ \\ $\dagger$ Department of Chemistry, Indian Institute of Technology-Kanpur, \\ Kanpur-208 016 , India . \\ $\S$ Present Address: Regional Research Laboratory, Trivandrum, \\ Kerala- 695 019, India. \\ ¥ Bruker India Scientific Pvt. Ltd., Lucknow 226022, India.
}

\section{Table of Contents}

S2 $\quad$ FAB mass spectrum of $\mathbf{2}$ and $\mathbf{3}$

S3 UV-vis spectrum of $\mathbf{3}$

S4 Temperature dependant ${ }^{1} \mathrm{H}$ NMR spectra of free base form of $\mathbf{2}$ in $\mathrm{CD}_{2} \mathrm{Cl}_{2}$.

S5 Open aperture Z-scan traces of heptaphyrins (6 and 7 in Ref.8c)

S6 Open aperture Z-scan trace of $\mathbf{3}$

S7 Synthetic procedure for core modified decaphyrin analogue $\mathbf{2}$ as a typical example.

S8 Optimized co-ordinates for core modified decaphyrin analogue 2. 
FAB-MASS Spectrum of 2:

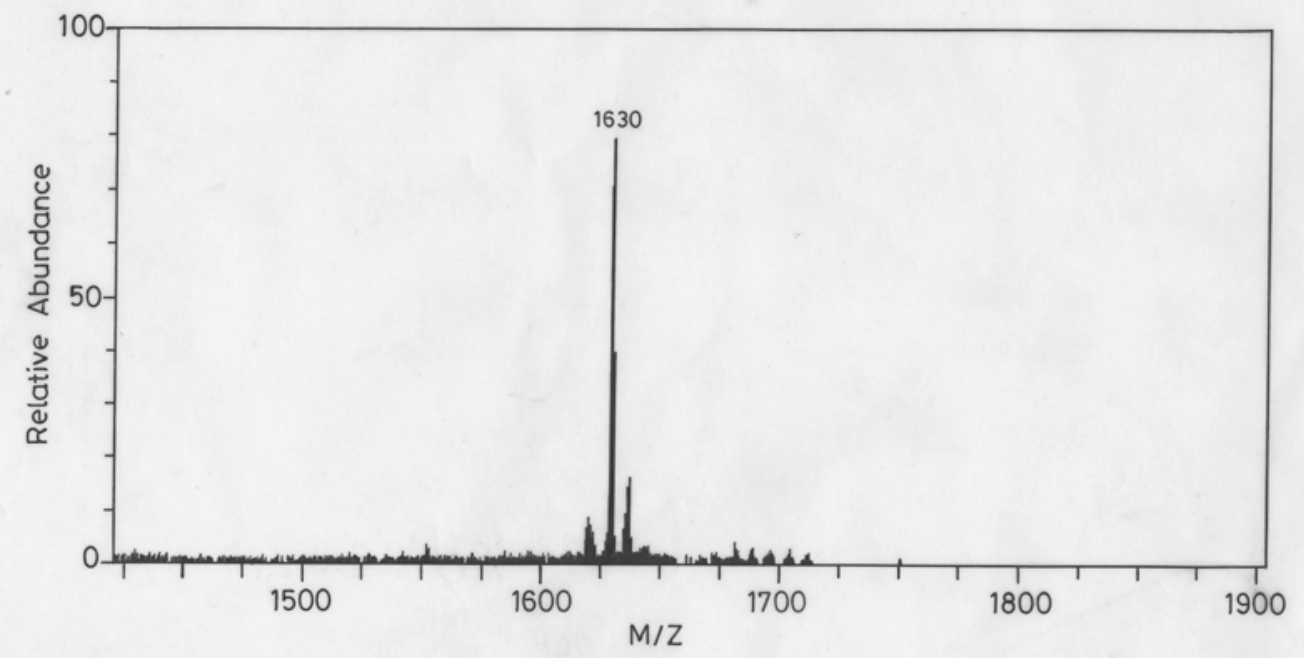

FAB-MASS Spectrum of 3 :

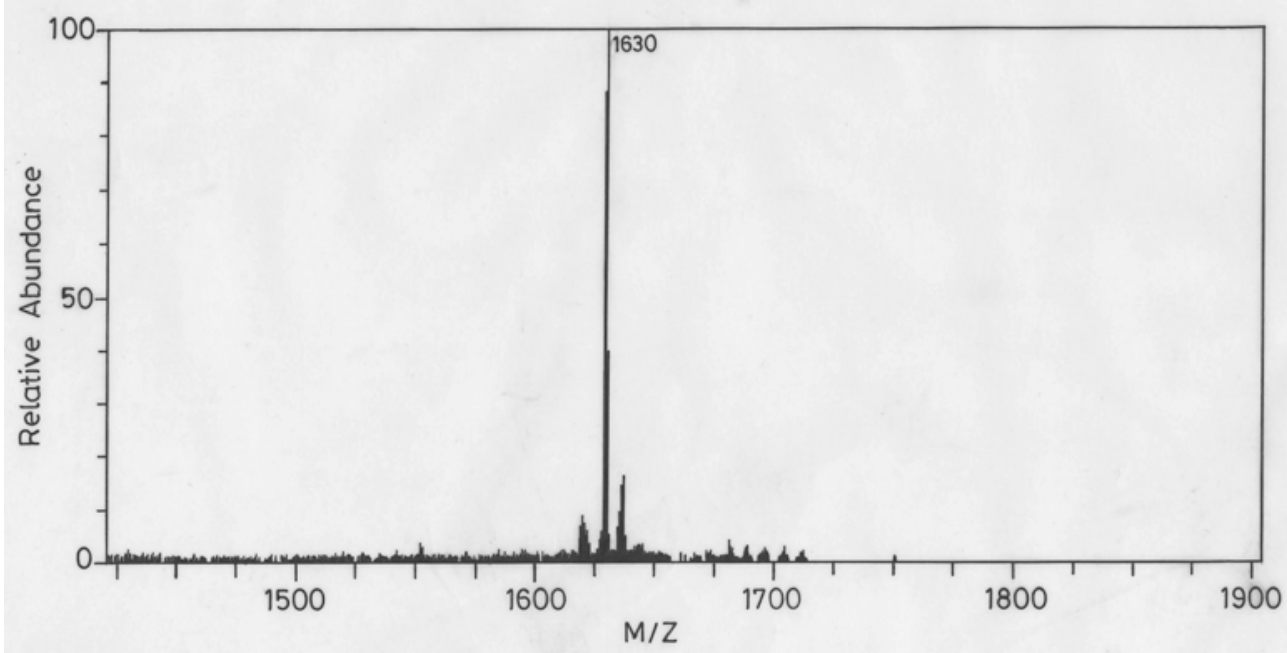


UV-Vis Spectrum of 3

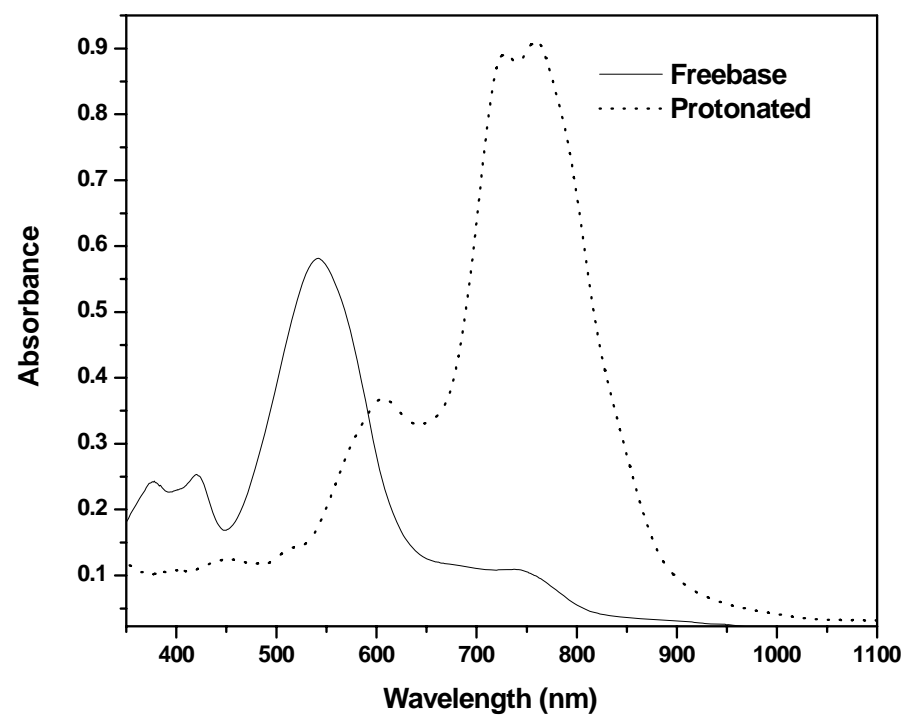


Temperature dependant ${ }^{1} H$ NMR spectra of free base form of 2 in $\mathrm{CD}_{2} \mathrm{Cl}_{2}$
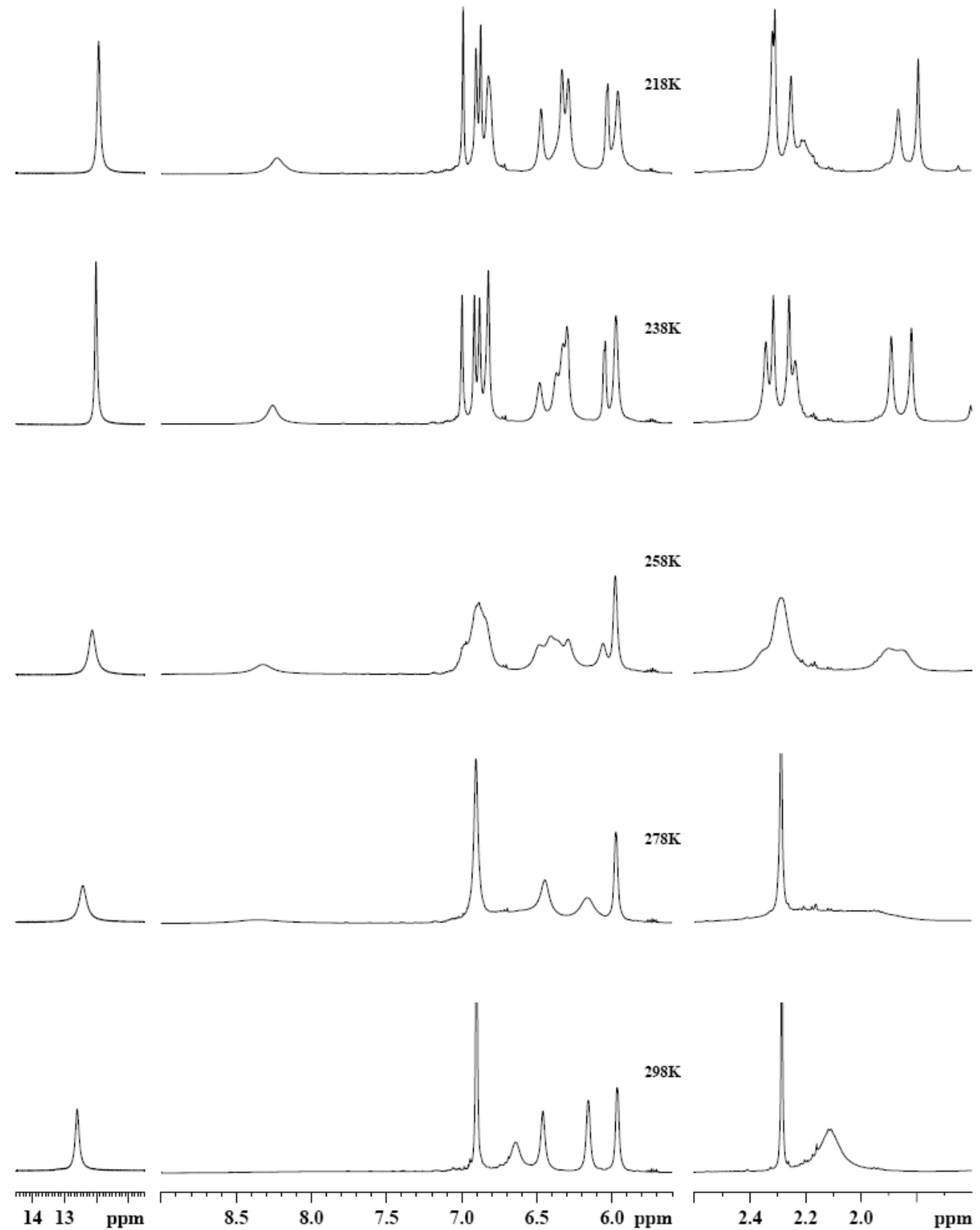
Open aperture Z-scan traces of heptaphyrins (6 and 7 in Ref. 8c)
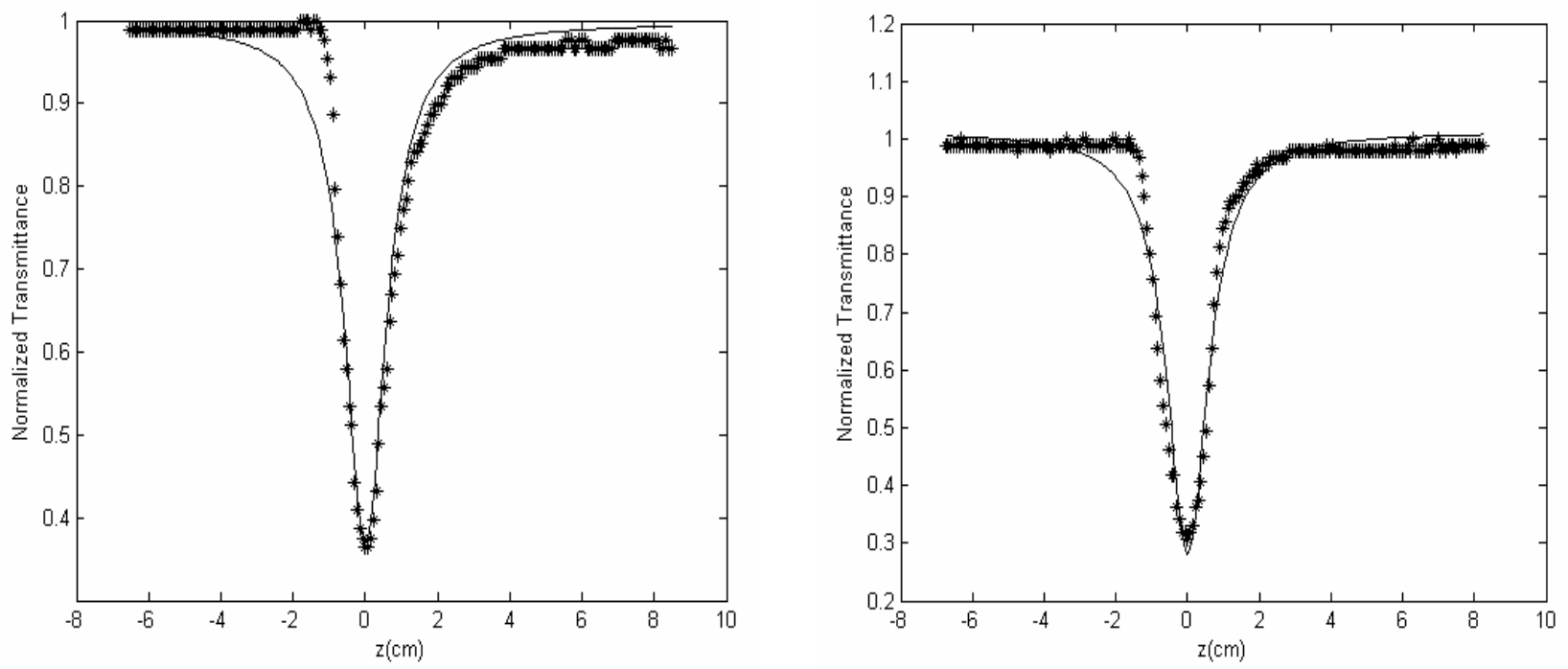

$\sigma_{2}=68,400 \mathrm{GM}$

$\sigma_{2}=78,600 \mathrm{GM}$ 
Open aperture Z-scan trace of 3

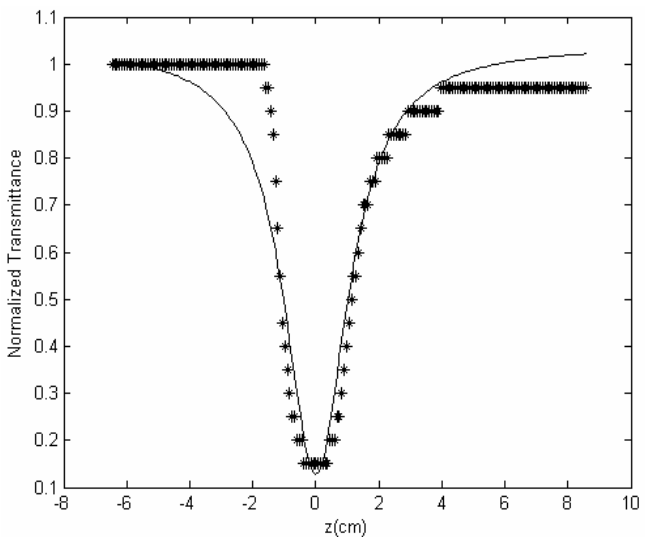


Synthetic procedure for core modified decaphyrin analogue 2 as a typical example.

In a typical procedure, modified pentapyrrane $\mathbf{1}$ $(0.6545 \mathrm{~g}, 1.02 \mathrm{mmol})$ was reacted with pentafluorobenzaldehyde $(0.12 \mathrm{ml}, 1.1 \mathrm{mmol})$ in dry dichloromethane $(250 \mathrm{~mL})$ and stirred under a nitrogen atmosphere in the absence of light for $15 \mathrm{~min} . p$-Tolyl sulphonic acid ( $0.0970 \mathrm{~g}, 0.55 \mathrm{mmol}) /$ methane sulphonic acid $(0.007 \mathrm{ml}, 0.11 \mathrm{mmol})$ was added and stirring continued for $9 \odot \mathrm{min}$. The reaction mixture was exposed to air, and chloranil $(0.2925 \mathrm{~g}, 1.1 \mathrm{mmol})$ was added; the reaction mixture was refluxed for $90 \mathrm{~min}$ on a preheated oil bath. The solvent was removed under reduced pressure. Upon purification by column chromatography with alumina (basic, grade III), a pink band eluted with $2: 3$ dichloromethane/petroleum ether, which on solvent evaporation afforded 2 as a dark green solid [168 mg, 11\%]. 


\title{
Optimized co-ordinates for core modified decaphyrin
}

\author{
analogue 2 .
}

\begin{tabular}{|c|c|c|c|c|c|}
\hline \multirow{2}{*}{$\begin{array}{l}\text { Center } \\
\text { Number }\end{array}$} & \multirow{2}{*}{$\begin{array}{l}\text { Atomic } \\
\text { Number }\end{array}$} & \multirow{2}{*}{$\begin{array}{l}\text { Atomic } \\
\text { Type }\end{array}$} & \multicolumn{3}{|c|}{ Coordinates (Angstroms) } \\
\hline & & & $\mathrm{x}$ & $\mathrm{Y}$ & Z \\
\hline 1 & 6 & $\Theta$ & -1.876418 & -2.882239 & 2.011425 \\
\hline 2 & 6 & $\odot$ & -2.100941 & -4.235651 & 1.597505 \\
\hline 3 & 6 & $\odot$ & $-\odot .974810$ & -4.977956 & 1.366100 \\
\hline 4 & 6 & $\odot$ & $\odot .258369$ & -4.283246 & 1.533986 \\
\hline 5 & 16 & $\odot$ & -0.063253 & -2.593160 & 2.157181 \\
\hline 6 & 1 & $\odot$ & -3.104371 & -4.628241 & 1.519917 \\
\hline 7 & 1 & 0 & -0.999876 & -6.010043 & 1.043718 \\
\hline 8 & 6 & 0 & 1.518652 & -4.728545 & 1.216510 \\
\hline 9 & 6 & $\odot$ & 1.928965 & -6.043244 & $\odot .863316$ \\
\hline 10 & 6 & $\odot$ & 3.261424 & -6.183856 & 0.560205 \\
\hline 11 & 6 & $\odot$ & 4.027516 & -4.987702 & $\odot .605965$ \\
\hline 12 & 16 & $\odot$ & 2.957904 & -3.598333 & 1.145003 \\
\hline 13 & 1 & $\odot$ & 1.228387 & -6.866723 & $\odot .85265 \odot$ \\
\hline 14 & 1 & 0 & 3.707713 & -7.124649 & ๑.269036 \\
\hline 15 & 6 & $\odot$ & 5.343966 & -4.793157 & $\odot .262488$ \\
\hline 16 & 6 & $\odot$ & 6.325841 & -5.779180 & -0.028496 \\
\hline 17 & 6 & $\odot$ & 7.567944 & -5.283418 & -0.333159 \\
\hline 18 & 6 & $\odot$ & 7.681056 & -3.859487 & -0.333827 \\
\hline 19 & 16 & 0 & 6.058739 & -3.112641 & ๑.080687 \\
\hline 20 & 1 & 0 & 6.105251 & -6.836948 & 0.022385 \\
\hline 21 & 1 & $\odot$ & 8.428993 & -5.896616 & -0.558149 \\
\hline 22 & 6 & $\odot$ & 8.822027 & -3.122358 & -0.570672 \\
\hline 23 & 6 & $\odot$ & -2.785800 & -1.897743 & 2.306262 \\
\hline 24 & 6 & $\odot$ & -6.273880 & -1.472075 & 1.565891 \\
\hline 25 & 6 & $\odot$ & -5.872717 & -2.281419 & $\odot .394259$ \\
\hline 26 & 6 & 0 & -4.575488 & -2.611231 & $\odot .581965$ \\
\hline 27 & 6 & $\odot$ & -4.155485 & -1.978672 & 1.861457 \\
\hline 28 & 7 & $\odot$ & -5.174904 & -1.334154 & 2.442480 \\
\hline 29 & 1 & $\odot$ & -6.511618 & -2.509843 & -0.436957 \\
\hline 30 & 1 & 0 & -1.041182 & -0.535813 & $\odot .438048$ \\
\hline 31 & 6 & 0 & 8.093761 & ๑.379846 & -0.546281 \\
\hline 32 & 6 & $\odot$ & 9.568323 & $\odot .444487$ & -0.575212 \\
\hline 33 & 6 & $\odot$ & 10.018188 & $-\odot .833854$ & -0.633090 \\
\hline 34 & 6 & $\odot$ & 8.820721 & -1.699100 & -0.596203 \\
\hline 35 & 7 & $\odot$ & 7.693566 & -0.960892 & -0.552053 \\
\hline 36 & 1 & $\odot$ & 10.150546 & 1.349919 & -0.587205 \\
\hline 37 & 1 & 0 & 11.035405 & -1.184241 & -0.683682 \\
\hline 38 & 6 & $\odot$ & $-\odot .851788$ & 2.152859 & -2.518927 \\
\hline 39 & 6 & $\odot$ & $-\odot .918530$ & 3.263281 & -1.701640 \\
\hline 40 & 6 & 0 & $\odot .308180$ & 3.643705 & -1.119782 \\
\hline 41 & 6 & $\odot$ & 1.386854 & 2.825284 & -1.419747 \\
\hline 42 & 16 & $\odot$ & $\odot .842143$ & 1.495954 & -2.558924 \\
\hline 43 & 1 & $\odot$ & -1.840722 & 3.808282 & -1.552657 \\
\hline 44 & 1 & $\odot$ & 3.105255 & 2.531080 & 1.980302 \\
\hline 45 & 6 & $\odot$ & -1.918888 & 1.527870 & -3.231924 \\
\hline
\end{tabular}




\begin{tabular}{|c|c|c|c|c|c|}
\hline 46 & 6 & 0 & -1.928589 & 0.850352 & -4.431405 \\
\hline 47 & 6 & 0 & -3.209013 & ๑. 390897 & -4.843833 \\
\hline 48 & 6 & 0 & -4.234377 & $\odot .678805$ & -3.970022 \\
\hline 49 & 16 & 0 & -3.594986 & 1.599865 & -2.530114 \\
\hline 50 & 1 & 0 & -1.029484 & $\odot .702072$ & -5.014298 \\
\hline 51 & 1 & 0 & -3.369356 & $-\odot .175171$ & -5.751183 \\
\hline 52 & 6 & 0 & -5.615909 & $\odot .319011$ & -3.990692 \\
\hline 53 & 6 & 0 & -6.480214 & $\odot .150231$ & -5.057213 \\
\hline 54 & 6 & 0 & -7.803154 & -0.167037 & -4.674721 \\
\hline 55 & 6 & 0 & -8.014469 & -0.279111 & -3.304419 \\
\hline 56 & 16 & 0 & -6.450496 & $\odot .005641$ & -2.407037 \\
\hline 57 & 1 & 0 & -6.181190 & $\odot .301885$ & -6.085386 \\
\hline 58 & 1 & 0 & -8.613552 & -0.297338 & -5.378740 \\
\hline 59 & 6 & 0 & -9.252859 & -0.525814 & -2.638359 \\
\hline 60 & 6 & 0 & 2.720051 & 2.991727 & -0.930448 \\
\hline 61 & 6 & 0 & 3.741561 & 2.071028 & $-\odot .985325$ \\
\hline 62 & 6 & 0 & 3.753368 & $\odot .651007$ & -1.382016 \\
\hline 63 & 6 & 0 & 5.027305 & $\odot .2 \odot 9752$ & -1.220880 \\
\hline 64 & 6 & 0 & 5.814877 & 1.368290 & $-\odot .748015$ \\
\hline 65 & 7 & 0 & 5.038092 & 2.451074 & -0.595979 \\
\hline 66 & 1 & 0 & 2.902662 & $\odot .065570$ & -1.686537 \\
\hline 67 & 1 & 0 & 5.425700 & $-\odot .776464$ & -1.369055 \\
\hline 68 & 6 & 0 & -8.518536 & -0.874071 & 0.775723 \\
\hline 69 & 6 & 0 & -9.988333 & -0.900319 & $\odot .890253$ \\
\hline 70 & 6 & 0 & -10.482265 & $-\odot .82404 \odot$ & $-\odot .381095$ \\
\hline 71 & 6 & 0 & -9.325169 & -0.714495 & -1.272331 \\
\hline 72 & 7 & 0 & -8.155456 & -0.762337 & $-\odot .509146$ \\
\hline 73 & 1 & 0 & -10.532658 & -1.030588 & 1.807181 \\
\hline 74 & 1 & 0 & -11.514045 & -0.833053 & $-\odot .691724$ \\
\hline 75 & 6 & $\odot$ & 7.234584 & 1.461231 & -0.561834 \\
\hline 76 & 6 & $\odot$ & -7.514971 & -0.941883 & 1.819341 \\
\hline 77 & 6 & 0 & 10.116983 & -3.846239 & $-\odot .803375$ \\
\hline 78 & 6 & 0 & 10.541118 & -4.113646 & -2.120653 \\
\hline 79 & 6 & $\odot$ & 11.749484 & -4.789411 & -2.321173 \\
\hline 80 & 6 & 0 & 12.545550 & -5.197358 & -1.245486 \\
\hline 81 & 6 & $\odot$ & 12.109091 & -4.920220 & $\odot .054368$ \\
\hline 82 & 6 & 0 & $10.9 \odot 4599$ & -4.249597 & ๑.293983 \\
\hline 83 & 6 & 0 & 9.695579 & -3.679000 & -3.305248 \\
\hline 84 & 1 & $\odot$ & 12.073362 & -5.000642 & -3.335473 \\
\hline 85 & 6 & 0 & 13.871173 & -5.899405 & -1.484676 \\
\hline 86 & 1 & $\odot$ & 12.716094 & -5.230949 & ๑.898961 \\
\hline 87 & 6 & 0 & 10.455249 & -3.957321 & 1.715400 \\
\hline 88 & 6 & 0 & 8.950027 & 4.450387 & 0.990975 \\
\hline 89 & 6 & 0 & 8.513575 & 3.152713 & 0.762307 \\
\hline $9 \odot$ & 6 & 0 & 7.762993 & 2.832529 & -0.366320 \\
\hline 91 & 6 & $\Theta$ & 7.471043 & 3.852364 & -1.269589 \\
\hline 92 & 6 & $\odot$ & 7.889432 & 5.153040 & -1.044874 \\
\hline 93 & 6 & 0 & 8.632267 & 5.452371 & ๑.087533 \\
\hline 94 & 6 & 0 & -1.765821 & ๑.401714 & 2.253429 \\
\hline 95 & 6 & $\odot$ & -1.312452 & 1.540403 & 2.928289 \\
\hline 96 & 6 & $\odot$ & -1.429444 & 1.665322 & 4.315555 \\
\hline 97 & 6 & 0 & -2.029968 & 0.625843 & 5.033626 \\
\hline 98 & 6 & $\odot$ & -2.492631 & -0.528746 & 4.395944 \\
\hline 99 & 6 & 0 & -2.341009 & -0.644868 & 3.001116 \\
\hline $10 \odot$ & 6 & $\odot$ & -1.657376 & 0.317432 & 0.740572 \\
\hline 101 & 1 & 0 & $-\odot .862066$ & 2.344572 & 2.35528 \\
\hline 102 & 6 & $\odot$ & $-\odot .948929$ & 2.921507 & 5.021773 \\
\hline
\end{tabular}




\begin{tabular}{|c|c|c|c|c|c|}
\hline 103 & 1 & $\odot$ & -2.142244 & $\odot .714148$ & 6.109651 \\
\hline 104 & 6 & $\odot$ & -3.196315 & -1.623509 & 5.175590 \\
\hline 105 & 6 & $\odot$ & 3.477399 & 5.395268 & -1.035415 \\
\hline 106 & 6 & $\odot$ & 3.666141 & 6.633231 & -0.411322 \\
\hline 107 & 6 & 0 & 3.396241 & 6.816631 & $\odot .948648$ \\
\hline 108 & 6 & $\odot$ & 2.942875 & 5.723395 & 1.694017 \\
\hline 109 & 6 & $\odot$ & 2.748167 & 4.468515 & 1.106826 \\
\hline 110 & 6 & $\odot$ & 3.002079 & 4.315241 & $-\odot .26940 \odot$ \\
\hline 111 & 6 & $\odot$ & 3.863456 & $5.20 \odot 219$ & -2.489633 \\
\hline 112 & 1 & $\odot$ & 4.034298 & 7.467341 & -1.000597 \\
\hline 113 & 6 & 0 & 3.629700 & 8.163562 & 1.611209 \\
\hline 114 & 1 & $\odot$ & 2.741520 & 5.845219 & 2.753712 \\
\hline 115 & 6 & $\odot$ & 2.309967 & 3.284653 & 1.951438 \\
\hline 116 & 6 & $\odot$ & -12.849806 & $-\odot .620132$ & -5.010581 \\
\hline 117 & 6 & $\odot$ & -12.171773 & -1.802085 & -4.694753 \\
\hline 118 & 6 & $\odot$ & -11.003764 & -1.792324 & -3.924580 \\
\hline 119 & 6 & $\odot$ & -10.502028 & $-\odot .558009$ & -3.466423 \\
\hline 120 & 6 & $\odot$ & -11.166259 & 0.646017 & -3.775889 \\
\hline 121 & 6 & $\odot$ & -12.333941 & $\odot .593842$ & -4.544221 \\
\hline 122 & 6 & $\odot$ & -14.135511 & $-\odot .655941$ & -5.818168 \\
\hline 123 & 1 & $\odot$ & -12.557260 & -2.751086 & -5.053720 \\
\hline 124 & 6 & $\odot$ & -10.290626 & -3.091764 & -3.590285 \\
\hline 125 & 6 & $\odot$ & -10.633353 & 1.976149 & -3.271725 \\
\hline 126 & 1 & $\odot$ & -12.848649 & 1.519365 & -4.781964 \\
\hline 127 & 6 & $\odot$ & -7.223656 & 1.393570 & 4.766017 \\
\hline 128 & 6 & $\odot$ & -8.214630 & $\odot .911715$ & 5.608549 \\
\hline 129 & 6 & 0 & -8.993187 & -0.167173 & 5.218572 \\
\hline 130 & 6 & 0 & -8.760857 & $-\odot .762728$ & 3.988200 \\
\hline 131 & 6 & $\odot$ & -7.769447 & -0.301422 & 3.133758 \\
\hline 132 & 6 & $\odot$ & -7.005367 & $\odot .793673$ & 3.533322 \\
\hline 133 & 1 & $\odot$ & -10.595057 & 1.987934 & -2.176598 \\
\hline 134 & 1 & $\odot$ & -9.617272 & 2.154572 & -3.640080 \\
\hline 135 & 1 & 0 & -11.274319 & 2.796850 & -3.605991 \\
\hline 136 & 1 & $\odot$ & -10.297022 & -3.269278 & -2.509046 \\
\hline 137 & 1 & $\odot$ & -10.782011 & -3.935208 & -4.083682 \\
\hline 138 & 1 & $\odot$ & -9.244479 & -3.059288 & -3.914403 \\
\hline 139 & 1 & 0 & -14.251656 & $\odot .256453$ & -6.411898 \\
\hline 140 & 1 & 0 & -14.148844 & -1.514722 & -6.496849 \\
\hline 141 & 1 & $\odot$ & -15.004340 & -0.738054 & -5.152312 \\
\hline 142 & 1 & $\odot$ & -3.242797 & -1.373454 & 6.239631 \\
\hline 143 & 1 & $\odot$ & -4.214136 & -1.731652 & 4.784057 \\
\hline 144 & 1 & $\odot$ & -2.678894 & -2.583512 & 5.064993 \\
\hline 145 & 1 & $\odot$ & -2.647346 & $\odot .201687$ & $\odot .284167$ \\
\hline 146 & 1 & 0 & -1.200011 & 1.226029 & $\odot .340031$ \\
\hline 147 & 1 & $\odot$ & -3.928801 & -3.158191 & $-\odot .083048$ \\
\hline 148 & 1 & $\odot$ & -1.745183 & 3.676721 & 5.048821 \\
\hline 149 & 1 & 0 & -0.655210 & 2.703147 & 6.053558 \\
\hline 150 & 1 & $\odot$ & $-\odot .088375$ & 3.356315 & 4.502262 \\
\hline 151 & 1 & $\odot$ & 4.005326 & 6.166136 & -2.983738 \\
\hline 152 & 1 & 0 & 4.801947 & 4.638147 & -2.541300 \\
\hline 153 & 1 & 0 & 3.098300 & 4.637317 & -3.034484 \\
\hline 154 & 1 & $\odot$ & 1.412840 & 2.804977 & 1.545411 \\
\hline 155 & 1 & $\odot$ & $\odot .426 \odot 59$ & 4.503851 & -0.477273 \\
\hline 156 & 1 & 0 & 2.096075 & 3.601636 & 2.976849 \\
\hline 157 & 1 & $\odot$ & 2.964230 & 8.299049 & 2.469980 \\
\hline 158 & 1 & $\odot$ & 4.664130 & 8.240630 & 1.970407 \\
\hline 159 & 1 & $\odot$ & 3.456362 & 8.983265 & $\odot .906327$ \\
\hline
\end{tabular}




\begin{tabular}{|c|c|c|c|c|c|}
\hline 160 & 1 & $\odot$ & 8.694067 & -4.120228 & -3.253475 \\
\hline 161 & 1 & $\odot$ & 9.574428 & -2.589875 & -3.316323 \\
\hline 162 & 1 & $\odot$ & 10.164625 & -3.985940 & -4.244685 \\
\hline 163 & 1 & $\odot$ & 11.209450 & -4.294304 & 2.432501 \\
\hline 164 & 1 & $\odot$ & 10.292341 & -2.883243 & 1.858418 \\
\hline 165 & 1 & $\odot$ & 9.510314 & -4.465140 & 1.938590 \\
\hline 166 & 1 & $\odot$ & 14.683925 & -5.166568 & -1.571016 \\
\hline 167 & 1 & $\odot$ & 14.112994 & -6.573995 & -0.657012 \\
\hline 168 & 1 & $\odot$ & 13.844859 & -6.483269 & -2.410431 \\
\hline 169 & 9 & $\odot$ & -9.549849 & -1.819112 & 3.611915 \\
\hline 170 & 9 & $\odot$ & -9.973271 & $-\odot .637222$ & 6.042182 \\
\hline 171 & 9 & $\odot$ & -8.430100 & $1.5 \odot 4077$ & 6.815909 \\
\hline 172 & 9 & $\odot$ & -6.475127 & 2.465305 & 5.151764 \\
\hline 173 & 9 & $\odot$ & -6.054639 & 1.309800 & 2.718217 \\
\hline 174 & 9 & $\odot$ & 8.833774 & 2.190941 & 1.672799 \\
\hline 175 & 9 & $\odot$ & 9.683671 & 4.745710 & 2.102219 \\
\hline 176 & 9 & $\odot$ & 9.053708 & 6.728231 & $\odot .308235$ \\
\hline 177 & 9 & $\odot$ & 7.577084 & 6.138429 & -1.934146 \\
\hline 178 & 9 & $\odot$ & 6.766400 & 3.598514 & -2.410378 \\
\hline
\end{tabular}

178

scf done: -7188.604978

$\begin{array}{lrrr}\text { C } & -1.876418 & -2.882239 & 2.011425 \\ \text { C } & -2.100941 & -4.235651 & 1.597505 \\ \text { C } & -0.974810 & -4.977956 & 1.366100 \\ \text { S } & 0.258369 & -4.283246 & 1.533986 \\ \text { H } & -0.063253 & -2.593160 & 2.157181 \\ \text { H } & -0.999871 & -4.628241 & 1.519917 \\ \text { C } & 1.518652 & -6.010043 & 1.043718 \\ \text { C } & 1.928965 & -6.043244 & 1.216510 \\ \text { C } & 3.261424 & -6.183856 & 0.863316 \\ \text { C } & 4.027516 & -4.987702 & 0.605965 \\ \text { S } & 2.957904 & -3.598333 & 1.145003 \\ \text { H } & 1.228387 & -6.866723 & 0.852650 \\ \text { H } & 3.707713 & -7.124649 & 0.269036 \\ \mathrm{C} & 5.343966 & -4.793157 & 0.262488 \\ \mathrm{C} & 6.325841 & -5.779180 & -0.028496 \\ \mathrm{C} & 7.567944 & -5.283418 & -0.333159 \\ \mathrm{C} & 7.681056 & -3.859487 & -0.333827 \\ \mathrm{~S} & 6.058739 & -3.112641 & 0.080687 \\ \mathrm{H} & 6.105251 & -6.836948 & 0.022385 \\ \mathrm{H} & 8.428993 & -5.896616 & -0.558149 \\ \mathrm{C} & 8.822027 & -3.122358 & -0.570672 \\ \mathrm{C} & -2.785800 & -1.897743 & 2.306262 \\ \mathrm{C} & -6.273880 & -1.472075 & 1.565891 \\ \mathrm{C} & -5.872717 & -2.281419 & 0.394259 \\ \mathrm{C} & -4.575488 & -2.611231 & 0.581965 \\ \mathrm{C} & -4.155485 & -1.978672 & 1.861457 \\ \mathrm{~N} & -5.174904 & -1.334154 & 2.442480 \\ \mathrm{H} & -6.511618 & -2.509843 & -0.436957 \\ \mathrm{H} & -1.041182 & -0.535813 & 0.438048 \\ \mathrm{C} & 8.093761 & 0.379846 & -0.546281 \\ \mathrm{C} & 9.568323 & 0.444487 & -0.575212 \\ \mathrm{C} & 10.018188 & -0.833854 & -0.633090 \\ \mathrm{C} & 8.820721 & -1.699100 & -0.596203\end{array}$




\begin{tabular}{|c|c|c|c|}
\hline $\mathrm{N}$ & 7.693566 & -0.960892 & -0.552053 \\
\hline $\mathrm{H}$ & 10.150546 & 1.349919 & -0.587205 \\
\hline $\mathrm{H}$ & 11.035405 & -1.184241 & -0.683682 \\
\hline C & -0.851788 & 2.152859 & -2.518927 \\
\hline C & $-\odot .918530$ & 3. 263281 & -1.701640 \\
\hline C & ๑ . 308180 & 3.643705 & -1.119782 \\
\hline C & 1.386854 & 2.825284 & -1.419747 \\
\hline$S$ & ๑. 842143 & 1.495954 & -2.558924 \\
\hline $\mathrm{H}$ & -1.840722 & 3.808282 & -1.552657 \\
\hline $\mathrm{H}$ & 3.105255 & 2.531080 & 1.980302 \\
\hline C & -1.918888 & 1.527870 & -3.231924 \\
\hline C & -1.928589 & ๑. 850352 & -4.431405 \\
\hline C & -3.209013 & ๑. 390897 & -4.843833 \\
\hline C & -4.234377 & ๑. 678805 & -3.970022 \\
\hline S & -3.594986 & 1.599865 & -2.530114 \\
\hline $\mathrm{H}$ & -1.029484 & ๑. 702072 & -5.014298 \\
\hline $\mathrm{H}$ & -3.369356 & -0.175171 & -5.751183 \\
\hline C & -5.615909 & 0. 319011 & -3.990692 \\
\hline C & -6.480214 & $\odot .150231$ & -5.057213 \\
\hline C & -7.803154 & -0.167037 & -4.674721 \\
\hline C & -8.014469 & -0.279111 & -3.304419 \\
\hline S & -6.450496 & ๑.005641 & -2.407037 \\
\hline $\mathrm{H}$ & -6.181190 & ๑. 301885 & -6.085386 \\
\hline $\mathrm{H}$ & -8.613552 & -0.297338 & -5.378740 \\
\hline C & -9.252859 & -0.525814 & -2.638359 \\
\hline C & 2.720051 & 2.991727 & -0.930448 \\
\hline C & 3.741561 & 2.071028 & -0.985325 \\
\hline C & 3.753368 & ๑. 651007 & -1.382016 \\
\hline C & 5.027305 & ๑. 209752 & -1.220880 \\
\hline C & 5.814877 & 1.368290 & -0.748015 \\
\hline $\mathrm{N}$ & 5.038092 & 2.451074 & -0.595979 \\
\hline $\mathrm{H}$ & 2.902662 & ๑. 065570 & -1.686537 \\
\hline $\mathrm{H}$ & 5.425700 & -0.776464 & -1.369055 \\
\hline C & -8.518536 & -0.874071 & 0.775723 \\
\hline C & -9.988333 & -0.900319 & 0.890253 \\
\hline C & -10.482265 & -0.824040 & -0.381095 \\
\hline C & -9.325169 & -0.714495 & -1.272331 \\
\hline $\mathrm{N}$ & -8.155456 & -0.762337 & -0.509146 \\
\hline $\mathrm{H}$ & -10.532658 & -1.030588 & 1.807181 \\
\hline $\mathrm{H}$ & -11.514045 & -0.833053 & -0.691724 \\
\hline C & 7.234584 & 1.461231 & -0.561834 \\
\hline C & -7.514971 & -0.941883 & 1.819341 \\
\hline C & 10.116983 & -3.846239 & -0.803375 \\
\hline C & 10.541118 & -4.113646 & -2.120653 \\
\hline C & 11.749484 & -4.789411 & -2.321173 \\
\hline C & 12.545550 & -5.197358 & -1.245486 \\
\hline C & 12.109091 & -4.920220 & 0.054368 \\
\hline C & 10.904599 & -4.249597 & 0.293983 \\
\hline C & 9.695579 & -3.679000 & -3.305248 \\
\hline $\mathrm{H}$ & 12.073362 & -5.000642 & -3.335473 \\
\hline C & 13.871173 & -5.899405 & -1.484676 \\
\hline $\mathrm{H}$ & 12.716094 & -5.230949 & 0.898961 \\
\hline C & 10.455249 & -3.957321 & 1.715400 \\
\hline C & 8.950027 & 4.450387 & 0.990975 \\
\hline C & 8.513575 & 3.152713 & 0.762307 \\
\hline C & 7.762993 & 2.832529 & -0.366320 \\
\hline C & 7.471043 & 3.852364 & -1.269589 \\
\hline
\end{tabular}




\begin{tabular}{|c|c|c|c|}
\hline C & 7.889432 & 5.153040 & -1.044874 \\
\hline ; & 8.632267 & 5.452371 & $\odot .087533$ \\
\hline ; & -1.765821 & $\odot .401714$ & 2.253429 \\
\hline C & -1.312452 & 1.540403 & 2.928289 \\
\hline C & -1.429444 & 1.665322 & 4.315555 \\
\hline C & -2.029968 & $\odot .625843$ & 5.033626 \\
\hline C & -2.492631 & $-\odot .528746$ & 4.395944 \\
\hline C & -2.341009 & $-\odot .644868$ & 3.001116 \\
\hline C & -1.657376 & $\odot .317432$ & $\odot .740572$ \\
\hline $\mathrm{H}$ & $-\odot .862066$ & 2.344572 & 2.355285 \\
\hline C & $-\odot .948929$ & 2.921507 & 5.021773 \\
\hline $\mathrm{H}$ & -2.142244 & $\odot .714148$ & 6.109651 \\
\hline C & -3.196315 & -1.623509 & 5.175590 \\
\hline C & 3.477399 & 5.395268 & -1.035415 \\
\hline C & 3.666141 & 6.633231 & -0.411322 \\
\hline C & 3.396241 & 6.816631 & $\odot .948648$ \\
\hline C & 2.942875 & 5.723395 & 1.694017 \\
\hline C & 2.748167 & 4.468515 & 1.106826 \\
\hline C & $3.0 \odot 2079$ & 4.315241 & $-\odot .26940 \odot$ \\
\hline C & 3.863456 & 5.200219 & -2.489633 \\
\hline $\mathrm{H}$ & 4.034298 & 7.467341 & -1.000597 \\
\hline C & 3.629700 & 8.163562 & 1.611209 \\
\hline $\mathrm{H}$ & 2.741520 & 5.845219 & 2.753712 \\
\hline C & 2.309967 & 3.284653 & 1.951438 \\
\hline C & -12.849806 & $-\odot .620132$ & -5.010581 \\
\hline C & -12.171773 & -1.802085 & -4.694753 \\
\hline C & -11.003764 & -1.792324 & -3.924580 \\
\hline C & -10.502028 & $-\odot .558009$ & -3.466423 \\
\hline C & -11.166259 & $\odot .646017$ & -3.775889 \\
\hline C & -12.333941 & $\odot .593842$ & -4.544221 \\
\hline C & -14.135511 & $-\odot .655941$ & -5.818168 \\
\hline $\mathrm{H}$ & -12.557260 & -2.751086 & -5.053720 \\
\hline C & -10.290626 & -3.091764 & -3.590285 \\
\hline C & -10.633353 & 1.976149 & -3.271725 \\
\hline $\mathrm{H}$ & -12.848649 & 1.519365 & -4.781964 \\
\hline C & -7.223656 & 1.393570 & 4.766017 \\
\hline C & -8.214630 & $\odot .911715$ & 5.608549 \\
\hline C & -8.993187 & $-\odot .167173$ & 5.218572 \\
\hline C & -8.760857 & $-\odot .762728$ & 3.988200 \\
\hline C & -7.769447 & -0.301422 & 3.133758 \\
\hline C & -7.005367 & $\odot .793673$ & 3.533322 \\
\hline $\mathrm{H}$ & -10.595057 & 1.987934 & -2.176598 \\
\hline $\mathrm{H}$ & -9.617272 & 2.154572 & -3.640080 \\
\hline $\mathrm{H}$ & -11.274319 & 2.796850 & -3.605991 \\
\hline $\mathrm{H}$ & -10.297022 & -3.269278 & $-2.509 \odot 46$ \\
\hline $\mathrm{H}$ & -10.782011 & -3.935208 & -4.083682 \\
\hline $\mathrm{H}$ & -9.244479 & -3.059288 & -3.914403 \\
\hline $\mathrm{H}$ & -14.251656 & ๑. 256453 & -6.411898 \\
\hline $\mathrm{H}$ & -14.148844 & -1.514722 & -6.496849 \\
\hline $\mathrm{H}$ & -15.004340 & -0.738054 & -5.152312 \\
\hline $\mathrm{H}$ & -3.242797 & -1.373454 & 6.239631 \\
\hline $\mathrm{H}$ & -4.214136 & -1.731652 & 4.784057 \\
\hline H & -2.678894 & -2.583512 & 5.064993 \\
\hline $\mathrm{H}$ & -2.647346 & ๑. 201687 & 0.284167 \\
\hline $\mathrm{H}$ & -1.200011 & 1.226029 & $\odot .340031$ \\
\hline $\mathrm{H}$ & -3.928801 & -3.158191 & $-\odot .083 \odot 48$ \\
\hline $\mathrm{H}$ & -1.745183 & 3.676721 & 5.048821 \\
\hline
\end{tabular}




$\begin{array}{rrrr}\text { H } & -0.655210 & 2.703147 & 6.053558 \\ \mathrm{H} & -0.088375 & 3.356315 & 4.502262 \\ \mathrm{H} & 4.005326 & 6.166136 & -2.983738 \\ \mathrm{H} & 4.801947 & 4.638147 & -2.541300 \\ \mathrm{H} & 3.098300 & 4.637317 & -3.034484 \\ \mathrm{H} & 1.412840 & 2.804977 & 1.545411 \\ \mathrm{H} & 0.426059 & 4.503851 & -0.477273 \\ \mathrm{H} & 2.096075 & 3.601636 & 2.976849 \\ \mathrm{H} & 2.964230 & 8.299049 & 2.469980 \\ \mathrm{H} & 4.664130 & 8.240630 & 1.970407 \\ \mathrm{H} & 3.456362 & 8.983265 & 0.906327 \\ \mathrm{H} & 8.694067 & -4.120228 & -3.253475 \\ \mathrm{H} & 9.574428 & -2.589875 & -3.316323 \\ \mathrm{H} & 10.164625 & -3.985940 & -4.244685 \\ \mathrm{H} & 11.209450 & -4.294304 & 2.432501 \\ \mathrm{H} & 10.292341 & -2.883243 & 1.858418 \\ \mathrm{H} & 9.510314 & -4.465140 & 1.938590 \\ \mathrm{H} & 14.683925 & -5.166568 & -1.571016 \\ \mathrm{H} & 14.112994 & -6.573995 & -0.657012 \\ \mathrm{H} & 13.844859 & -6.483269 & -2.410431 \\ \mathrm{~F} & -9.549849 & -1.819112 & 3.611915 \\ \mathrm{~F} & -9.973271 & -0.637222 & 6.042182 \\ \mathrm{~F} & -8.430100 & 1.504077 & 6.815909 \\ \mathrm{~F} & -6.475127 & 2.465305 & 5.151764 \\ \mathrm{~F} & -6.054639 & 1.309800 & 2.718217 \\ \mathrm{~F} & 8.833774 & 2.190941 & 1.672799 \\ \mathrm{~F} & 9.683671 & 4.745710 & 2.102219 \\ \mathrm{~F} & 9.053708 & 6.728231 & 0.308235 \\ \mathrm{~F} & 7.577084 & 6.138429 & -1.934146 \\ \mathrm{~F} & 6.766400 & 3.598514 & -2.410378\end{array}$

